\title{
A low-cost and versatile system for projecting wide-field visual stimuli within fMRI scanners
}

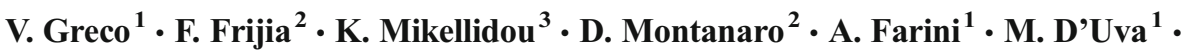 \\ P. Poggi ${ }^{1}$ - M. Pucci ${ }^{1}$ - A. Sordini ${ }^{1}$ • M. C. Morrone ${ }^{3}$ - D. C. Burr ${ }^{4,5,6}$
}

Published online: 20 June 2015

(C) Psychonomic Society, Inc. 2015

\begin{abstract}
We have constructed and tested a custom-made magnetic-imaging-compatible visual projection system designed to project on a very wide visual field $\left(\sim 80^{\circ}\right)$. A standard projector was modified with a coupling lens, projecting images into the termination of an image fiber. The other termination of the fiber was placed in the 3-T scanner room with a projection lens, which projected the images relayed by the fiber onto a screen over the head coil, viewed by a participant wearing magnifying goggles. To validate the system, widefield stimuli were presented in order to identify retinotopic visual areas. The results showed that this low-cost and versatile optical system may be a valuable tool to map visual areas in the brain that process peripheral receptive fields.
\end{abstract}

Keywords Wide-field retinotopy $\cdot$ fMRI $\cdot$ Visual projection system

Functional magnetic resonance imaging (fMRI) is currently one of the most widely used noninvasive techniques for measuring local neural activity. Most fMRI studies involve projecting

D. C. Burr

dave@in.cnr.it

1 National Institute of Optics, National Research Council, Florence, Italy

2 Fondazione CNR/Regione Toscana G. Monasterio, Pisa, Italy

3 Department of Translational Research on New Technologies in Medicine and Surgery, University of Pisa, Pisa, Italy

4 Department of Neuroscience, Psychology, Pharmacology and Child Health, University of Florence, Florence, Italy

5 Institute of Neuroscience, National Research Council, Pisa, Italy

6 Stella Maris Scientific Institute, Pisa, Italy visual stimuli using magnet-safe liquid crystal display or projectors equipped with custom optics that allow the delineation of visual brain areas. However, these techniques are compromised by their narrow fields of view $(<30 \mathrm{deg})$, thus limiting studies of the visual system to the central visual field. Consequently, the study of peripheral vision in both healthy and clinical human subjects, as well as the essential preoperative mapping of the entire visual field in associative cortices, is impossible with most projection systems available at present.

Two different types of 2-D visual stimulus presentation systems have been developed in the past. The first system (Roby, Gao, \& Fox, 2000) is based on back-projection and comprises an LCD projector with a modified lens (located outside the scanner room), a custom lens, a rear-projection screen, and an adjustable folding mirror. The last two components are mounted on the head coil of the scanner. The custom lens images the LCD of the projector on the rear-projection screen, and the participant views the stimuli on the screen through the adjustable folding mirror. The resulting field of view is $26 \times 56 \mathrm{deg}$. The second type, devised by Cornelissen, Pelli, Farell, and Huckins (1997) is based on a fiberscope. It comprises an LCD located outside the scanner room, the image fiber into which an objective lens projects, and an eyepiece through which the other end of the image fiber projects, thus allowing the participant to view the stimuli. The field of view achieved in this case is still limited, only $28 \times 35 \mathrm{deg}$.

In an attempt to increase the field of view, Pitzalis et al. (2006) implemented a few changes to a "traditional" visual presentation system. They used a custom head coil in a 1.5-T Siemens Vision MR scanner (Siemens Medical Systems, Erlangen, Germany), which could accommodate a $260 \times 185 \mathrm{~mm}$ back-projection screen. This screen was placed in the scanner at a distance of $100-120 \mathrm{~mm}$ from the participant's eyes and was viewed directly, rather than via a folding mirror, resulting in a $100 \times 80 \mathrm{deg}$ field of view. This setup, however, has a number of drawbacks: 
(a) It is not adaptable to other types of MRI scanners and head coils, because the back-projection screen is very large; (b) an expensive custom lens replaces the standard lens of the video projector; and (c) the distance between the video projector and the back-projection screen is limited to 3-4 m.

A further increase of the field of view was achieved by Arnoldussen et al. (2011), who used a custom-made eightchannel occipital surface coil in a 3-T trio Siemens scanner (Siemens Medical Systems, Erlangen, Germany). Visual stimuli were projected, via a mirror, onto a small projection screen $(143 \times 80.5 \mathrm{~mm})$ by an LCD projector with $1,280 \times 1,024$ pixel resolution and custom-built optics. The screen was placed approximately $30 \mathrm{~mm}$ directly above the participant's eyes, and the projected stimuli were viewed through contact lenses of approximately +30 diopters. Although the field of view was $\sim 120^{\circ} \times 90^{\circ}$, this setup suffers from the same limitations as the previously described setup (Pitzalis et al., 2006), with an additional potentially limiting factor-that the participant has to use contact lenses.

Recently, Wu et al. (2013) developed a novel wide-view visual presentation system that is adaptable to all types of scanners. They used a Mitsubishi LVP-HC6800 projector (1, $600 \times 1,200$ pixels, $60 \mathrm{~Hz}$ ), whose standard lens was replaced with a 70 - to $300-\mathrm{mm}$ focal length Nikon camera zoom lens, to back-project stimuli $(460 \times 425$ pixels $)$ onto a hemispheric screen through a folding mirror placed on the head coil. The concave spherical screen $(52 \mathrm{~mm}$ in diameter, radius of curvature $30 \mathrm{~mm}$ ) was placed $30 \mathrm{~mm}$ away from the participant's eyes, and the participant viewed the images through contact lenses between +20 and +25 diopters. The resulting field of view was increased to $120^{\circ} \times 120^{\circ}$. However, this setup still has some drawbacks, because the video projector has to be relatively near the fMRI scanner (3-4 m), and the participants must use contact lenses.

In addition, Yan, Jin, $\mathrm{He}$, and $\mathrm{Wu}$ (2011) and $\mathrm{Wu}$ et al. (2013) used an optical fiber bundle of large diameter, equal to $52 \mathrm{~mm}$ (8,700 elements), and replaced the eyepiece with a contact lens. The end face of the fiber bundle was a concave spherical surface $30 \mathrm{~mm}$ in radius. By choosing the power of the contact lens to be between +20 and +25 diopters, they could place the spherical face of the fiber bundle a distance of $30 \mathrm{~mm}$ from the eye of the participant, increasing the field of view to $120^{\circ} \times 120^{\circ}$. However, this setup also has some drawbacks: (a) Because of its large diameter, the fiber bundle is bulky, heavy, stiff, and of limited length (4-5 m), and (b) the participants again must wear contact lenses.

In this article, we present a new 2-D visual stimulus presentation system obtained from the synthesis of the two types described above. Its main features are its low cost and versatility. In addition, it can display low-spatial-frequency components $(<0.02 \mathrm{c} / \mathrm{deg})$ onto a screen $80 \mathrm{deg}$ wide at $120 \mathrm{~Hz}$. It is adaptable to all types of scanners and head coils, and its optical setup eliminates many of the drawbacks of the two types mentioned above. Below we show the application of this new visual stimulus presentation system with a GE 3-T scanner (Excite HDx, GE Medical Systems, Milwaukee, WI), equipped with a standard eight-channel head coil. We confirmed its suitability for fMRI studies of visual perception by evaluating the cortical BOLD response of healthy human participants using two kinds of retinotopic stimuli. The stimuli projected were $115 \mathrm{~mm}$ in diameter, corresponding to approximately an 80-deg field of view and a gamma-calibrated mean luminance of $5 \mathrm{~cd} / \mathrm{m}^{2}$.

\section{Description of the visual projection system}

The 2-D visual presentation system described in this article is shown schematically in Fig. 1. It comprises an LCD video projector, a coupling lens (L1), an image fiber, a projection lens (L2), a mirror fixed at 45 degrees above a back-projection screen, and eyeglasses. A computer generates the stimuli on the LCD of the video projector, whose standard lens was removed. The coupling lens L1, placed just outside the video projector, projects the images shown on the LCD onto the termination of the image fiber. The other end of the image fiber is placed inside the scanner room, where it transmits through the projection lens L2 onto the folding mirror, and subsequently onto the back-projection screen. The mirror along with the back-projection screen is placed over the head coil inside the scanner bore, and the participant views the stimuli on the back-projection screen using the eyeglasses.

The LCD video projector (EH-TW5910, Epson, Long Beach, CA) was slightly modified by replacing its standard lens with an identical barrel, without lenses and spacers. To prevent dust entering the optical unit of the video projector, a glass window with an antireflective coating was placed at the top of the barrel. This empty barrel is necessary to funnel the cooling air into the video projector, to prevent overheating the LCD. The coupling lens (L1) is necessary to modify slightly the optical unit of the video projector, which normally produces a highly magnified image of the LCD $\left(\sim 10 \times 5.6 \mathrm{~mm}^{2}\right)$ on a projection screen (e.g., $2 \times 1.125 \mathrm{~m}^{2}$ ), to enable it instead to project a very reduced image onto the fiber termination (0.46-mm diameter). Because of this reduction, other objects located within the video projector are also imaged on the fiber termination, degrading the image of the LCD. When the video projector enlarges the image, these objects become completely out of focus on the projection screen. To eliminate this problem, we inserted diffusing elements in the optical path before the LCD.

L1 is an off-the-shelf, high-quality lens designed for megapixel cameras (effective focal length $=12 \mathrm{~mm}$; f-number $=$ $\mathrm{f} / 1.4$ ). It is located just outside the video projector, a distance of $160 \mathrm{~mm}$ from the LCD. Its purpose is to form a reduced 


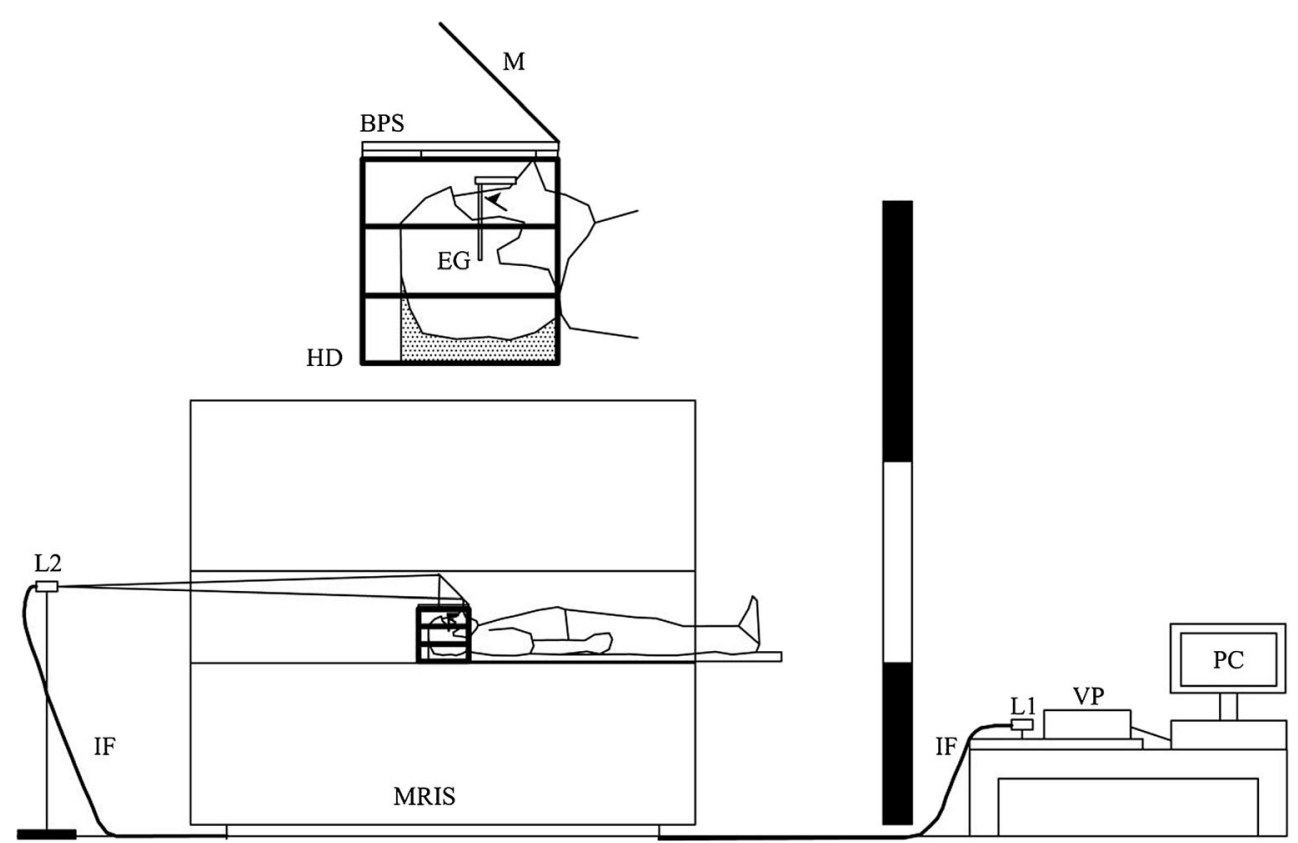

Fig. 1 Schematic drawing of the 2-D visual stimulus presentation system described in this article: (PC) personal computer, (VP) LCD video projector, (L1) coupling lens, (IF) image fiber, (L2) projection lens, (MRIS)
MRI scanner, (HD) head coil, (BPS) back-projection screen, (M) 45-deg mirror, (EG) eyeglasses image (magnification 0.082) of the LCD at the termination of the fiber, which is placed in the vicinity of its focal point.

The image fiber (FIGH-10-500N, Fujikura, Koto-ku, Japan) used has the following characteristics: 10,000 picture elements, 0.46-mm image circle diameter, 4.6- $\mu \mathrm{m}$ picture element diameter, pure silica glass core material, numerical aperture 0.22 , minimum bending radius $50 \mathrm{~mm}$, length $17 \mathrm{~m}$. Its purpose was to relay the image of the LCD formed by L1 into the scanner room. The number of picture elements defines the spatial resolution of the entire visual stimulus presentation system as $100 \times 100$ pixels. This value is greater than that used by Yan et al. (2011; 8,700 picture elements), but much less than the spatial resolution of the LCD itself $(1,920 \times 1,080$ pixels). However, since the focus of our investigation is on peripheral vision, this relatively low spatial resolution is adequate. An image fiber with 10,000 picture elements has additional benefits, since it can be very long $(17 \mathrm{~m})$, very flexible (minimum bending radius $50 \mathrm{~mm}$ ), very thin, and very lightweight (less than $1 \mathrm{~kg}$, including a further protective sheath). With current technology, increasing the number of the picture elements would cause a decrease in the maximum length and flexibility of the image fiber. The spatial resolution of the 2-D visual stimulus presentation system is only limited by the manufacturing technology of the image fiber; future improvements of the manufacturing technology would allow an increase of the spatial resolution of our custom-made setup.

The fiber termination, located outside the scanner room, must be positioned exactly at the focus of L1. Otherwise the fiber might project only a portion of the LCD, or even miss it completely. For this reason, the mechanical support of the fiber is equipped with a micrometric adjustment system.

Similarly to L1, L2 is an off-the-shelf high-quality lens (effective focal length $=8 \mathrm{~mm}$; f-number $=\mathrm{f} / 1.4$ ). As is shown in Fig. 1, it is located inside the scanner room. For this reason, L2 was initially completely disassembled and its mechanical parts (e.g., barrel, spacers) were replaced with exact copies made of PVC. The fiber termination located inside the scanner room must be positioned near the focus of L2. However, this positioning is less critical than with L1, because these highquality lenses are well-corrected over a wide field of view. In this case, the support of the fiber is only equipped with an axial micrometric adjustment to focus.

The diameter of the image formed by L2 on the backprojection screen varies linearly with the distance between the two. In our case, by applying the relevant equation, it is easily shown that

$\Delta=17.39 D+8$

where $\Delta$ denotes the distance in millimeters between L2 and the back-projection screen, and $D$ is the diameter (in millimeters) of the image formed on the back-projection screen. So, to have an image on the back-projection screen with a diameter of $110 \mathrm{~mm}$, L2 should be positioned at a distance of 1,921 mm from the back-projection screen. This value of $\Delta$ is reasonable and generally compatible with the dimensions of all scanners and of the rooms in which they are placed. 
The participant views the stimuli focused by L2 on the back-projection screen from inside the head coil. Due to the small distance $(\sim 75 \mathrm{~mm})$ between the back-projection screen and the participant's eye, the participant has to wear glasses to see the images clearly. These glasses are made entirely of plastic with power $+8,+10$, and +12 diopters, depending on the capacity of accommodation of the participant's eye. Commercial CR-39 plastic lenses with antireflection coating are enough to have a clear view of the stimuli projected on the screen.

The maximum size of the stimuli projected on the backprojection screen visible by the participant inside the head coil is limited by the size of the folding mirror and the design of the head coil. In practice, the maximum dimensions of the folding mirror are set by the size of the scanner bore, as is shown in Fig. 1. Moreover, because the head coils are designed to be more comfortable, they have structures that correspond to the nose and temples of the participant. For example, in the case of the scanner and the head coil considered in this article, by suitably shaping the folding mirror and the back-projection screen and varying $\Delta$, we could maximize the area that the participant could observe with one of the two eyes. This area had dimensions of $115 \mathrm{~mm}$ (left-right) $\times 82 \mathrm{~mm}$ (foreheadchin). This slight asymmetry was due to the fact that in one direction the folding mirror and in the other the head coil limited the size.

To calculate the field of view subtended at the eye by the viewed stimuli, it is necessary to consider the magnification introduced by the eyeglasses. Looking at the diagram in Fig. 2 and applying the relevant equations, it is easy to derive the following expressions:

$\mathrm{m}=\frac{\mathrm{D}^{\prime}}{\mathrm{D}}=\frac{1}{1-\mathrm{T} \cdot \Phi / 1000}$

and

$\mathrm{FOV}=2 \tan ^{-1}\left(\frac{1}{2} \frac{\mathrm{D}}{\mathrm{T}+\mathrm{t}-\mathrm{T} \cdot \mathrm{t} \cdot \Phi / 1000}\right)$

where $m$ is the magnification introduced by the eyeglasses, $D$ is the diameter of the stimuli projected on the back-projection screen by L2 (in mm), $D^{\prime}$ is the diameter of the stimuli seen through the eyeglasses (in millimeters), $T$ (in millimeters) is the distance between the back-projection screen and the eyeglasses, $\Phi$ (in diopters) is the power of the eyeglasses, and $t$ is the distance between the eyeglasses and the first nodal point of the eye (in millimeters). The latter distance is given by the sum of the vertex distance $(\sim 15 \mathrm{~mm})$ with the distance between the cornea and the first nodal point of the eye $(\sim 7 \mathrm{~mm})$. In our case, calculating Eq. 1 and Eq. 2 by using averages of $T=$

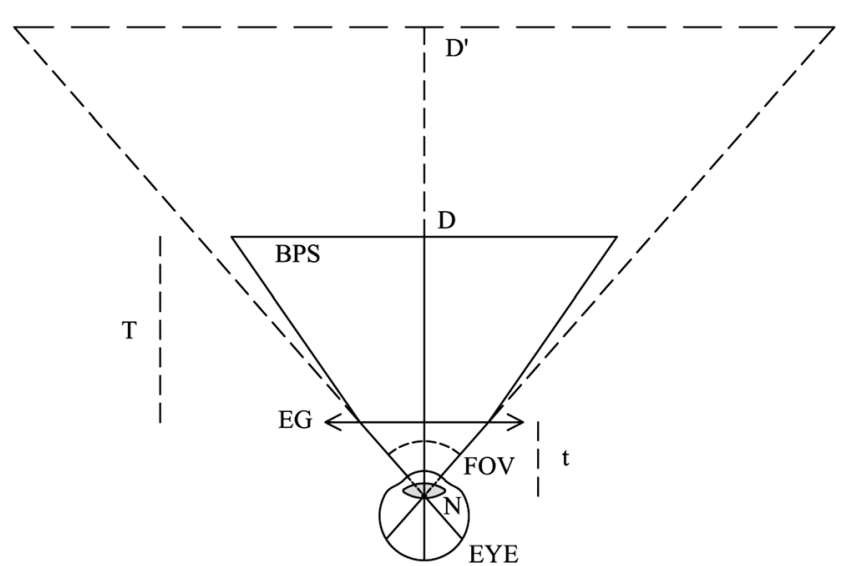

Fig. 2 Schematic drawing for the calculation of the field of view subtended at the eye of the participant: (EG) eyeglasses, (BPS) backprojection screen, (D) diameter of the stimuli, (D') apparent diameter of stimuli viewed through the eyeglasses, (T) distance between the BPS and the EG, $(\mathrm{t})$ distance between the EG and the first nodal point $(\mathrm{N})$ of the eye, (FOV) field of view subtended at the eye

$61 \mathrm{~mm}, t=22 \mathrm{~mm}$, and $\Phi=10$ diopters, it is evident that the eyeglasses introduce a magnification of 2.1 , and the $115 \times$ $82 \mathrm{~mm}$ area of the back-projection screen thus corresponds to an average wide field of view of $79^{\circ} \times 61^{\circ}$.

The setup shown in Fig. 1 does not require any modification of the MRI scanner. Only the detachable mirror is removed from the head coil of the scanner, and in its place, using the same mount, is mounted the custom-made support that holds the 45-deg mirror and the back-projection screen. However, thanks to the versatility of this 2-D visual stimulus presentation system, other setups are possible. For example, by modifying the custom-made support, the projection lens can be mounted near the feet of the participant on the scanner bed, and the image fiber can be put in the cable chain of the scanner. In this way, the projection lens moves along with the bed, which allows adjustments to be made while the participant is still outside the scanner.

\section{Evaluation of the visual projection system}

\section{fMRI scanning}

Scanning was performed with a GE 3-T scanner (Excite HDx, GE Medical Systems, Milwaukee, WI) at the Fondazione CNR/Regione Toscana G. Monasterio in Pisa, Italy. The study was approved by the ethics committee of the Azienda Ospedaliero-Universitaria Pisana (protocol number 3255, approved on 20/01/2009) and was in accordance with the ethical standards of the 1964 Declaration of Helsinki.

Six healthy participants (four male, two female; $25-$ 58 years old, all right-handed) with normal or corrected-tonormal visual acuity were scanned. Informed written consent was obtained from each participant prior to scanning sessions, 
in accordance with the guidelines of the MRI Laboratory. Each fMRI session consisted of two functional and one structural scans. Three-dimensional (3-D) anatomical images were acquired at a resolution of $1 \times 1 \times 1 \mathrm{~mm}$ using a T1-weighted, magnetization-prepared, fast spoiled gradient echo sequence $(\mathrm{FOV}=256 \mathrm{~mm}, \mathrm{BW}=15.63,256 \times 256$ matrix, $\mathrm{TE}=$ minimum full). An echo planar imaging sequence was used for the fMRI data acquisition $(\mathrm{FOV}=240 \mathrm{~mm}, 128 \times 128$ matrix, slice thickness $=3 \mathrm{~mm}, 19$ axial slices, flip angle $=90$, $\mathrm{TE}=30 \mathrm{~ms}, \mathrm{TR}=2,500 \mathrm{~ms}$ ). The first $13 \mathrm{~s}$ of each functional acquisition were discarded from the data analysis to achieve a steady state.

\section{Visual stimuli}

Stimuli were generated outside the scanner room using MATL AB software (VSG 2/5 Visual Stimulus Generator, The Mathworks, Natick, MA) in conjunction with routines from the Psychophysics Toolbox (Brainard, 1997). All participants viewed the stimuli monocularly, with the left eye covered by an eye patch.

Figure 3A displays the two types of stimuli used. To delineate the visual areas, the stimuli comprised 100 circular dots, half black and half white, moving on a gray background in two symmetrical sectors across the fixation point along the two principal meridians (Engel, Glover, \& Wandell, 1997; Sereno et al., 1995; Wandell, Brewer, \& Dougherty, 2005). A block design was used with meridians stimulated interchangeably for $15 \mathrm{~s}$ and the motion direction inverting seven times to avoid BOLD adaptation. Each block was repeated six times. Each dot had a lifetime of 20 frames, or $333 \mathrm{~ms}$ at a refresh rate of $60 \mathrm{~Hz}$. Locally, the dots moved along linear trajectories at a constant speed of $6.5 \%$ s.

To map the eccentricity of the visual cortex, we presented three annuli at different eccentricities, within which black and white checks reversed contrast at $4 \mathrm{~Hz}$. A block design was
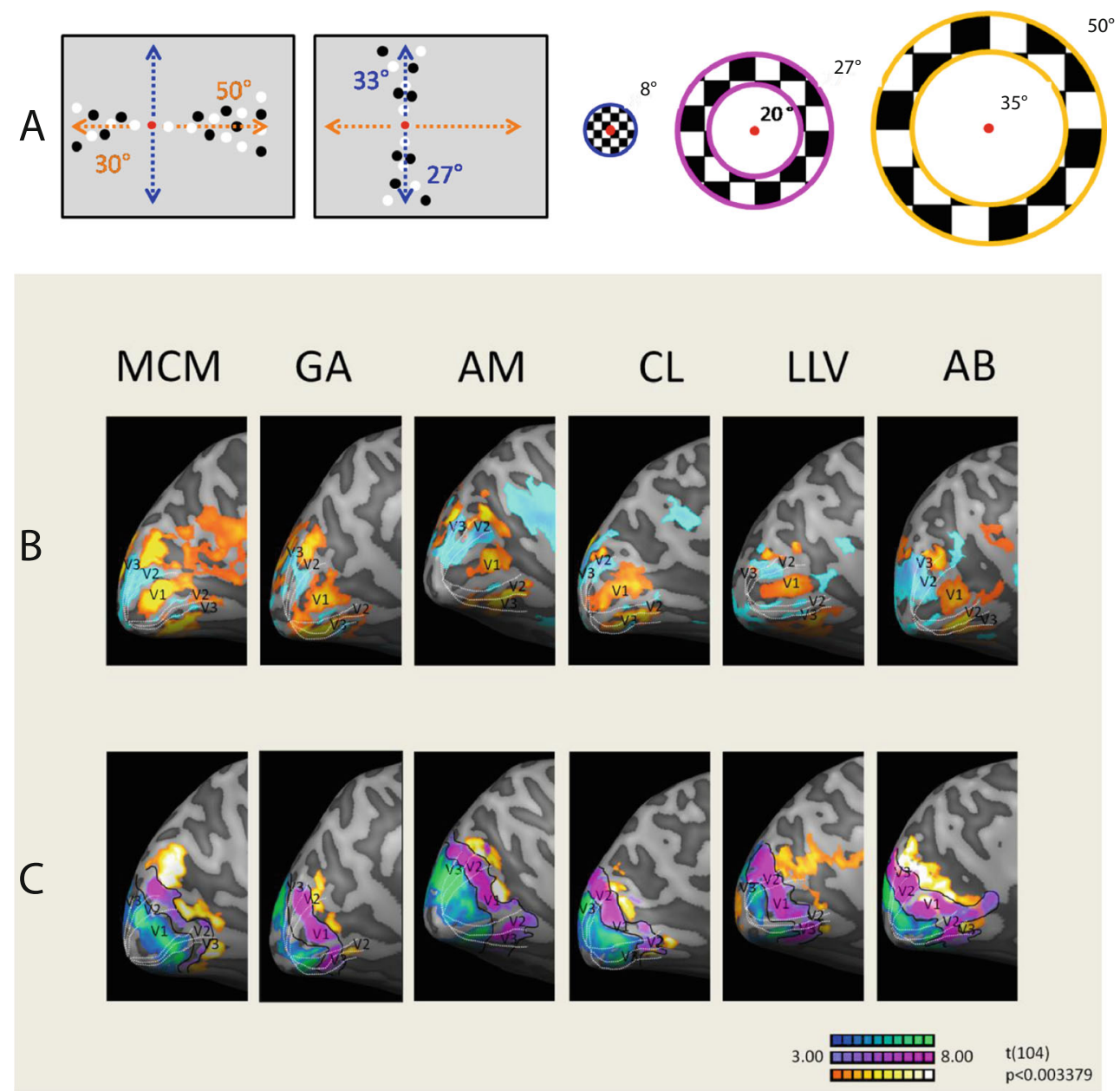

Fig. 3 (A) The two types of stimuli used to delineate visual areas. Note that all stimuli were black and white; the color coding is for clarity. (B, C) Medial views of the left hemispheres of six participants: (B) Responses to stimulation along the vertical meridian, in blue and cyan, and responses to

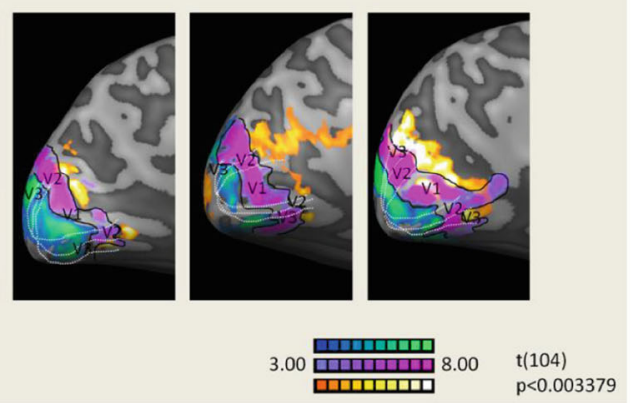

stimulation along the horizontal meridian, in orange and yellow. (C) Responses to stimuli at three different eccentricities; foveal is shown in blue and green, near eccentricity in purple, and far eccentricity in orange and yellow 
used, with each annulus being presented sequentially for $15 \mathrm{~s}$ starting with the most eccentric one, and each block was repeated six times. The outermost annulus had an outer radius of $50^{\circ}$ and an inner radius of $35^{\circ}$ of visual angle. The mediumsized annulus had an outer radius of $27^{\circ}$ and an inner radius of $20^{\circ}$, and the foveal annulus an outer radius of $8^{\circ}$ of visual angle.

\section{Data analysis}

The data analysis was carried out using Brain Voyager QX (Version 2.8, Brain Innovation, Maastricht, the Netherlands) and MATLAB (The Mathworks, Natick, MA). Prior to the statistical analysis, the functional data underwent preprocessing steps, including 3-D motion correction, linear trend removal, and high-pass filtering. Slice scan time correction was performed for the functional data. For each individual participant, the anatomical and functional data were transformed first into his or her own AC-PC space (rotating the cerebrum into the anterior commissure-posterior commissure plane), and then into Talairach space. To generate inflated surfaces for each hemisphere, the white-gray boundary was traced using an automatic segmentation algorithm, supplemented by manual correction by an expert operator to correct errors generated by the automatic routine. This segmentation was also used to automatically reconstruct the surface of the outer gray-matter boundary, which was subsequently inflated.

\section{Results}

The functional data were analyzed by fitting the hemodynamic response with a general linear model to the BOLD time series. Figure 3B shows the left-hemisphere inflated surfaces for six participants in response to meridian stimulation using wide-field stimuli in the horizontal dimension.

Figure $3 \mathrm{C}$ (second row) shows maps on a medial view of the cortical mesh of visual responses to an expanding ring, with color-coding indicating eccentricity (distance from the center of the visual field). A systematic increase in eccentricity $\left(0-8^{\circ}, 20-27^{\circ}, 35-50^{\circ}\right)$ is observed, moving anteriorly along the medial wall of the occipital cortex. As the expanding ring stimulus moved from the peripheral to the central portion of the retina, the corresponding cortical activations varied from the anterior to the posterior portions of the calcarine sulcus in the retinotopic map. The larger peripheral representation crossed to the fundus of the parieto-occipital sulcus.

\section{Discussion}

Our aim was to develop a robust and affordable optical system for projecting wide-field visual stimuli at $120 \mathrm{~Hz}$ within fMRI scanners, which would be useful for both neuroimaging research and key clinical functions, such as the mapping of tumors. Using flexible optic fibers, we can display any arbitrary image from a standard LCD projector outside the scanner room onto a back-projection screen approximately $8 \mathrm{~cm}$ away from the participant's eyes.

The main advantage of the present system over conventional ones is the size of the visual field: $80 \mathrm{deg}$ wide, as compared to the 30-deg field of view normally achieved using conventional goggles and backprojection systems. A field of this size is fundamental to many research goals, including studies of motion perception, and flow motion in particular; the assessment of visual areas with very large receptive fields, such as V6; and spatiotopy. More importantly, the new system also has many potential clinical applications, including mapping the effects of trauma or stroke in the occipital cortex, preparation for surgery in or near visual areas, and evaluating the benefits of vision restoration therapy through visual field changes.

We have previously shown that it is possible to record from alert human infants as young as 6 weeks old while the mother or an experimenter holds the infant in the scanner (Morrone, Biagi, Crespi, \& Tosetti, 2014). This projector system would be ideal for many studies - such as for mapping the visual brain areas in infants, which are still unknown-because it is large enough not to require accurate positioning. The display would be particularly useful for traditional recording from young anesthetized infants, whose gaze is hard to control and align.

Although the stimulated visual field is less than that in previous studies (Pitzalis et al., 2006; Wu et al., 2013; Yan et al., 2011), our projector has some advantages over the previous systems. First, it is adaptable and works in all commercial scanners, not only in the Vision MR scanner, which is currently used in very few laboratories and clinics. Second, the projector can be quite distant from the MR scanner, giving flexibility in stimulus generation. Third, the participants do not need to wear contact lenses, which can often cause eye irritation. Finally, and importantly, our system is relatively low-cost and versatile. Advances in technology also should lead to improvements in the system. For instance, developments in the production of optic fibers should allow for a substantial increase of the spatial resolution of the system, enough that it could be used to study central as well as peripheral vision. With improvements in projector lighting technology, it should be possible to increase the luminance of the stimuli two- or four-fold. Built in eye movement recording would also be valuable and would be relatively easy to implement. Perhaps the simplest way to accomplish this would be to add another fiber-optic bundle with a suitable lens to image the eye (under infrared illumination) onto a standard eyetracker outside the scanner. 


\section{References}

Arnoldussen, D. M., Goossens, J., \& van den Berg, A. V. (2011). Adjacent visual representations of self-motion in different reference frames. Proceedings of the National Academy of Sciences, 108, 11668-11673. doi:10.1073/pnas.1102984108

Brainard, D. H. (1997). The psychophysics toolbox. Spatial Vision, 10, 433-436. doi:10.1163/156856897X00357

Cornelissen, F. W., Pelli, D. G., Farell, B., \& Huckins, S. C. (1997). A binocular fiberscope for presenting visual stimuli during fMRI. Spatial Vision, 11, 75-81.

Engel, S., Glover, G., \& Wandell, B. (1997). Retinotopic organization in human visual cortex and the spatial precision of functional MRI. Cerebral Cortex, 7, 181-192.

Morrone, M. C., Biagi, L., Crespi, S., \& Tosetti, M. (2014). Development of visual BOLD response in infants. Journal of Vision, 14(10), 14. doi: $10.1167 / 14.10 .14$

Pitzalis, S., Galletti, C., Huang, R. S., Patria, F., Committeri, G., Galati, G., ... Sereno, M. (2006). Wide-field retinotopy defines human cortical visual area V6. Journal of Neuroscience, 26, 7962-7973. doi:10.1523/jneurosci.0178-06.2006
Roby, J. W., Gao, J. H., \& Fox, P. (2000). A versatile, low-cost method for presenting visual stimuli during MRI. Journal of Magnetic Resonance Imaging, 11, 223-227. doi:10.1002/(sici)15222586(200002)11:2<223::aid-jmri22>3.0.co;2-g

Sereno, M., Dale, A., Reppas, J., Kwong, K., Belliveau, J., Brady, T., ... Tootell, R. (1995). Borders of multiple visual areas in humans revealed by functional magnetic resonance imaging. Science, 268, 889-893. doi:10.1126/science.7754376

Wandell, B., Brewer, A., \& Dougherty, R. F. (2005). Visual field map clusters in human cortex. Philosophical Transactions of the Royal Society B, 360, 693-707. doi:10.1098/rstb.2005. 1628

Wu, J., Wang, B., Yang, J. J., Hikino, Y., Takahashi, S., Yan, T., ... Kanazawa, S. (2013). Development of a method to present wideview visual stimuli in MRI for peripheral visual studies. Journal of Neuroscience Methods, 214, 126-136. doi:10.1016/j.jneumeth. 2013.01.021

Yan, T., Jin, F., He, J., \& Wu, J. (2011). Development of a wide-view visual presentation system for visual retinotopic mapping during functional MRI. Journal of Magnetic Resonance Imaging, 33, 441-447. doi:10.1002/jmri.22404 